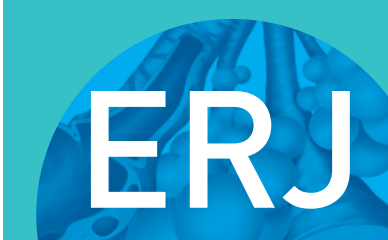

open research
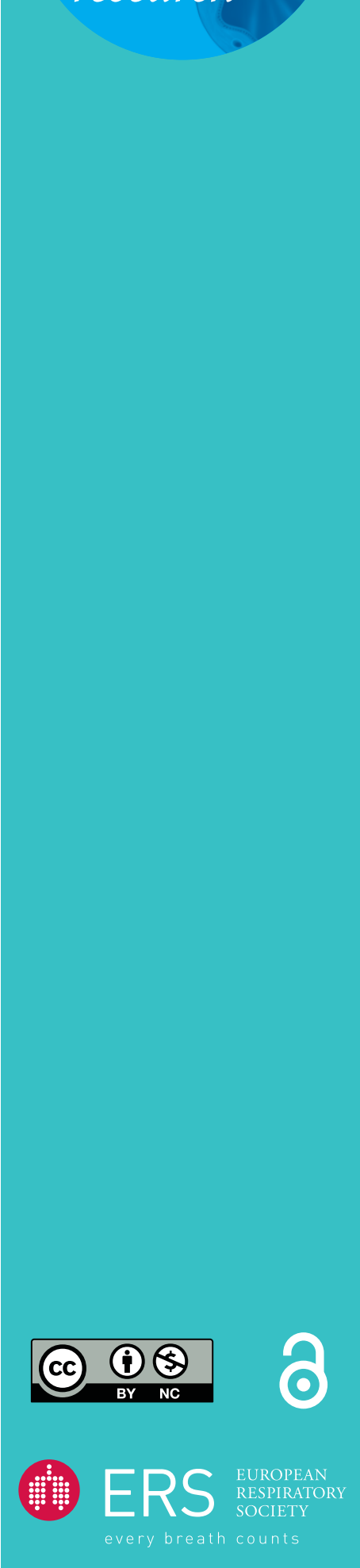

\section{Seasonal variation in asthma exacerbations in the AUSTRI and VESTRI studies}

\section{To the Editor:}

Seasonal peaks in asthma exacerbations are well described, as is the age-dependent nature of the magnitude of these peaks [1]. Children with asthma experience seasonal peaks in exacerbation frequency, which vary according to geographical location and climate [1-3]. Previous studies have examined the effect of season on the efficacy of pharmacological interventions. The anti-immunoglobulin-E monoclonal antibody omalizumab showed an increase in treatment benefits compared with placebo in the autumn and spring versus summer months in children, adolescents and young adults with allergic asthma in the USA [4]. In contrast, the anti-interleukin-5 monoclonal antibody mepolizumab showed no effect of seasonal differences in adolescents and adults with severe eosinophilic asthma [5].

To further investigate the seasonal effects of treatment on asthma exacerbations, we performed a post hoc analysis of two large asthma clinical studies: one in children (VESTRI; clinicaltrials.gov NCT01462344) and another in adults/adolescents (AUSTRI; clinicaltrials.gov NCT01475721). These safety studies evaluated the risk of a serious asthma-related event following administration of the inhaled corticosteroid (ICS) fluticasone propionate (FP) in combination with the long-acting $\beta_{2}$-agonist (LABA) salmeterol (FP/ SAL) in patients with persistent asthma, compared with FP alone [6, 7].

VESTRI was an international, randomised, double-blind, 26-week trial that evaluated the safety and efficacy of FP/SAL versus FP alone in 6208 children [6]. VESTRI included patients (aged 4-11 years) with a diagnosis of asthma who had consistently used asthma medication during the 4 weeks preceding study entry and had a history of asthma exacerbations during the previous 12 months. No numerically higher risk of composite safety end-point events (death, endotracheal intubation and hospitalisation (all asthma-related); primary end-point) was observed with FP/SAL versus FP (HR 1.28; 95\% CI 0.73-2.27). Patients receiving FP/SAL had numerically fewer severe asthma exacerbations than those receiving FP alone (HR 0.86; 95\% CI 0.73-1.01) [6].

AUSTRI was an international, randomised, double-blind, 26-week trial conducted in 11679 patients with asthma (11\% were adolescents) [7]. Eligible patients had a diagnosis of persistent asthma which required daily medication for asthma control, and a history of $\geqslant 1$ severe asthma exacerbation in the year prior to study entry. The primary end-point was non-inferiority between FP/SAL and FP alone on the composite safety end-point. Patients receiving FP/SAL did not have a numerically higher risk of composite safety endpoint events than those receiving FP alone (HR for the time-to-event analysis $1.03,95 \%$ CI 0.64-1.66) [7]. Fewer patients receiving FP/SAL had a severe asthma exacerbation versus those receiving FP alone (HR 0.79, 95\% CI 0.70-0.89; $\mathrm{p}<0.001$ ) [7].

In this post hoc analysis of VESTRI and AUSTRI, the outcome of interest was the seasonal incidence of severe asthma exacerbations and the seasonal variation in the effect of the addition of SAL to FP. A severe exacerbation was defined as an asthma deterioration that required glucocorticoid treatment for a minimum of 3 days (both studies), or a hospital or emergency department visit that was asthma related and led to the use of systemic glucocorticoids (AUSTRI), or a depot injection of glucocorticoids (VESTRI) [6, 7].

@ERSpublications

Seasonal variation in the benefit of LABA/ICS versus ICS on asthma exacerbation rate is observed in children. http://ow.ly/pcZF30o8hHk

Cite this article as: Szefler SJ, Raphiou I, Zeiger RS, et al. Seasonal variation in asthma exacerbations in the AUSTRI and VESTRI studies. ERJ Open Res 2019; 5: 00153-2018 [https:// doi.org/10.1183/23120541.00153-2018].

Copyright $\odot$ ERS 2019. This article is open access and distributed under the terms of the Creative Commons Attribution Non-Commercial Licence 4.0. 
Enrolment in both studies took 2-3 years, with a comparable number of subjects exposed in each season (autumn: $n=4542$ and $n=8463$; winter: $n=4204$ and $n=7526$; spring: $n=4567$ and $n=8202$; and summer: $\mathrm{n}=4501$ and $\mathrm{n}=8422$ for VESTRI and AUSTRI, respectively).

Summaries of population characteristics were based on the intent-to-treat population, comprising all randomised patients who received $\geqslant 1$ dose of study drug $[6,7]$. Efficacy data were collected only until discontinuation of the study drug; as such, efficacy analyses are presented using the modified intent-to-treat population. The relative risk of exacerbations per season was based on the proportion of patients reporting $\geqslant 1$ exacerbation during that season. Seasons were defined based on calendar month and the hemisphere and country in which the data were recorded. An asthma exacerbation was assigned to the season in which the onset of the exacerbation occurred. As such, a patient with an asthma exacerbation onset in more than one season would be counted as having an asthma exacerbation in each of those seasons. Anonymised individual participant data and study documents can be requested for further research from www.clinicalstudydatarequest.com

In VESTRI, the percentage of patients in the FP alone arm who experienced an exacerbation was $4.43 \%$ in autumn, $4.86 \%$ in winter, $3.72 \%$ in spring and $2.17 \%$ in summer (figure $1 \mathrm{a}$ ). In two of the four seasons, there were numerically fewer patients with an event in the FP/SAL arm than the FP arm $(\mathrm{p}=0.019$ in the winter (autumn: RR 0.79, 95\% CI 0.59-1.05; winter: RR 0.70, 95\% CI 0.52-0.95; spring: RR 0.99, 95\% CI 0.73-1.32; and summer: RR 1.11, 95\% CI 0.76-1.63)).

The percentage of patients in the FP alone arm in AUSTRI who experienced an exacerbation was 4.66\%, $5.12 \%, 3.30 \%$ and $2.96 \%$ in autumn, winter, spring and summer, respectively (figure $1 \mathrm{~b}$ ). In autumn and winter, there were fewer events $(\mathrm{p}=0.004$ and $\mathrm{p}=0.034$, respectively) in the FP/SAL arm than the FP arm (autumn: RR 0.74, 95\% CI 0.60-0.91; winter: RR 0.80, 95\% CI 0.65-0.98; spring: RR 0.89, 95\% CI 0.7-1.14; and summer: RR 0.83, 95\% CI 0.64-1.07).

In both studies, there was a seasonal effect on the severe exacerbation rate, with higher rates observed in autumn and winter compared with spring and summer. The beneficial effects of FP/SAL versus FP on exacerbation risk in children (VESTRI) had a marked seasonal difference, with a $30 \%$ risk reduction in
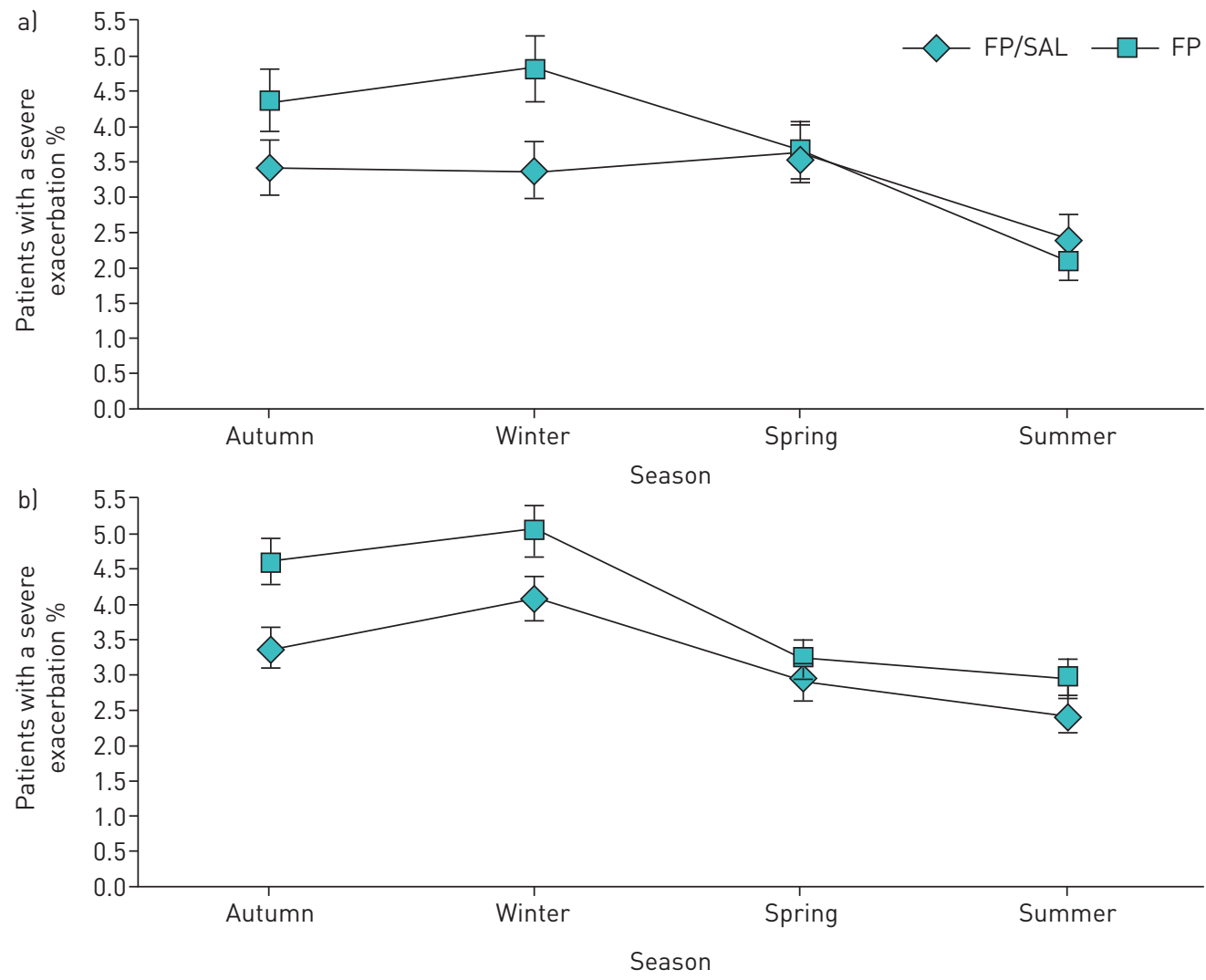

FIGURE 1 Proportion of patients with asthma experiencing a severe exacerbation by season in the a) VESTRI and b) AUSTRI clinical studies. Bars depict standard error. FP: fluticasone propionate; SAL: salmeterol. 
winter. In AUSTRI, there was a $26 \%$ and $20 \%$ reduced exacerbation risk favouring FP/SAL versus $\mathrm{FP}$ in the autumn and winter, respectively.

The seasonal variation in the treatment benefit seen in VESTRI has similarities to that observed for omalizumab in children with asthma during autumn and spring [4]. Taken together, these findings suggest that some pharmacological interventions may have enhanced effects during some of the seasons. These data have implications for the design of future studies, highlighting the need to include allowances for potential seasonal effects.

Reasons for the observed seasonal effects can only be the subject of speculation, but may be related in part to the increase in exacerbations seen during autumn and winter and the greater opportunities to observe effects of medication during those seasons. Evidence has previously suggested that ICS/LABA provides significantly greater protection against virally induced asthma exacerbations than ICS alone [8].

A limitation of these analyses is the use of a 6-month treatment period rather than a 12-month period, which would have spanned all seasons for each participant. Additionally, climatological differences, seasonal patterns and weather variabilities exist between some of the countries ( 31 in VESTRI and 33 in AUSTRI) and their regions (spanning North America, Europe, Latin America, South Africa, Asia and Australia) covered by the studies [6,7]; however, the proportion of patients from countries with variable seasonal and weather patterns was small and is unlikely to have affected the overall outcomes. A further limitation is the lack of spirometry and biomarker measurements to further assess the effect of treatment.

In summary, the present post hoc analysis of two large safety studies suggests that the combination of ICS/ LABA plays a role in reducing the seasonal variation in severe exacerbation rates in children and adults compared with ICS alone. Previous studies that averaged exacerbation rates over all seasons are likely to have underestimated treatment benefits in winter and overestimated benefits in summer. In patients who have had an exacerbation in the prior year, a step-up approach from ICS to ICS/LABA may be effective for reducing the risk of an exacerbation in the following year. Alternatively, if there is a history of a seasonal exacerbation in prior years, a step-up approach with ICS/LABA could be started prior to that season.

Stanley J. Szefler ${ }^{1}$, Ibrahim Raphiou ${ }^{2}$, Robert S. Zeiger ${ }^{3}$, David Stempel ${ }^{4}$, Kenneth Kral $^{2}$ and Steven Pascoe ${ }^{5}$

${ }^{1}$ Dept of Pediatrics, Breathing Institute, Children's Hospital Colorado and University School of Medicine, Aurora, CO, USA. ${ }^{2}$ GlaxoSmithKline, Research Triangle Park, NC, USA. ${ }^{3}$ Dept of Allergy and Research and Evaluation, Kaiser Permanente Southern California Region, San Diego and Pasadena, CA, USA. ${ }^{4}$ Propeller Health, San Francisco, CA, USA. ${ }^{5}$ GlaxoSmithKline, Upper Merion, PA, USA.

Correspondence: Steven Pascoe, GlaxoSmithKline, 709 Swedeland Road, King of Prussia, PA 19406, USA. E-mail: pascoesteve@yahoo.co.uk

Received: Sept 072018 | Accepted after revision: Feb 252019

Acknowledgements: Medical writing support in the form of developing/editing drafts based on author input, editorial assistance, and submission of the final manuscript was provided by Katy Tucker (Fishawack Indicia Ltd., Abingdon, UK), funded by GlaxoSmithKline.

Conflict of interest: S.J. Szefler reports other funding and non-financial support from GlaxoSmithKline, during the conduct of the study; other funding from Boehringer-Ingelheim, Genentech, GlaxoSmithKline, Aerocrine, Novartis, AstraZeneca, Daiichi Sankyo, Roche, and Teva, and grants from GlaxoSmithKline, outside the submitted work I. Raphiou reports other funding and non-financial support from GlaxoSmithKline during the conduct of the study; and personal fees and other funding from GlaxoSmithKline, outside the submitted work. R.S. Zeiger reports other funding and non-financial support from GlaxoSmithKline, during the conduct of the study; and grants from Aerocrine, Genentech, MedImmune/AstraZeneca, ALK, Merck and NHLBI, and personal fees from AstraZeneca, Genentech, Novartis, TEVA, GlaxoSmithKline, Theravance BioPharma, Sanofi, Regeneron Pharmaceuticals and Patara Pharma, outside the submitted work. D. Stempel reports other funding and non-financial support from GlaxoSmithKline, during the conduct of the study; and personal fees and other funding from GlaxoSmithKline, outside the submitted work. $\mathrm{K}$. Kral reports other funding and non-financial support from GlaxoSmithKline, during the conduct of the study; and personal fees and other funding from GlaxoSmithKline, outside the submitted work. S. Pascoe reports other funding and non-financial support from GlaxoSmithKline, during the conduct of the study; and personal fees and other funding from GlaxoSmithKline, outside the submitted work.

Support statement: This study was funded by GlaxoSmithKline. Funding information for this article has been deposited with the Crossref Funder Registry.

\section{References}

1 Gerhardsson de Verdier M, Gustafson P, McCrae C, et al. Seasonal and geographic variations in the incidence of asthma exacerbations in the United States. J Asthma 2017; 54: 818-824. 
2 Wisniewski JA, McLaughlin AP, Stenger PJ, et al. A comparison of seasonal trends in asthma exacerbations among children from geographic regions with different climates. Allergy Asthma Proc 2016; 37: 475-481.

3 Larsen K, Zhu J, Feldman LY, et al. The annual September peak in asthma exacerbation rates. Still a reality? Ann Am Thorac Soc 2016; 13: 231-239.

4 Busse WW, Morgan WJ, Gergen PJ, et al. Randomized trial of omalizumab (anti-IgE) for asthma in inner-city children. N Engl J Med 2011; 364: 1005-1015.

5 Ortega H, Chupp G, Bardin P, et al. The role of mepolizumab in atopic and nonatopic severe asthma with persistent eosinophilia. Eur Respir J 2014; 44: 239-241.

6 Stempel DA, Szefler SJ, Pedersen S, et al. Safety of adding salmeterol to fluticasone propionate in children with asthma. N Engl J Med 2016; 375: 840-849.

7 Stempel DA, Raphiou IH, Kral KM, et al. Serious asthma events with fluticasone plus salmeterol versus fluticasone alone. N Engl J Med 2016; 374: 1822-1830.

8 Prazma CM, Kral KM, Gul N, et al. Controller medications and their effects on asthma exacerbations temporally associated with upper respiratory infections. Respir Med 2010; 104: 780-787. 